и нехирургических методов в ортодонтической практике при закрытии постэкстракционных пространств: систематический обзор

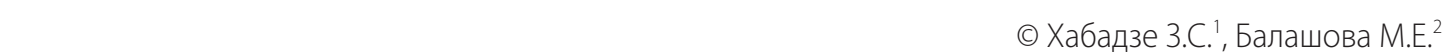

'Федеральное государственное автономное образовательное учреждение высшего образования «Российский университет дружбы народов», Москва, Россия

²Федеральное государственное бюджетное учреждение «Национальный медицинский исследовательский центр стоматологии и челюстно-лицевой хирургии» Министерства здравоохранения Российской Федерации, Москва, Россия

\begin{abstract}
Резюме:
Сокращение продолжительности ортодонтического лечения является важным вопросом, особеннодлявзрослых пациентов, которые хотят быстрее завершить лечение в виду социальных или эстетических обстоятельств. Кроме того, увеличение продолжительности ортодонтического лечения имеет ряд побочных эффектов, поэтому и ортодонты, и пациенты заинтересованы в применении методик, способных ускорить перемещение зубов. Сокращение сроков лечения может быть достигнуто путем сочетания ортодонтического и хирургических или нехирургических методов.

Цель. Систематическая оценка научных данных об эффективности и побочных эффектах хирургических и нехирургических методов в ускорении ортодонтического лечения в ситуациях с удалением премоляров и последующим закрытием постэкстракционных пространств.

Материалы и методы. Электронный поиск статей проводился с помощью поисковых систем и баз данных: Cyberleninka, eLIBRARY, Google Scholar, Pub Med, SCOPUS. Критерий даты публикации был выбран с января 2010 г. по январь 2021 г. Статьи, включенные в систематический обзор, должны были соответствовать следующим критериям включения: контролируемые клинические испытания и рандомизированные контролируемые клинические испытания, проведенные на людях и опубликованные в течение последних 11 лет на английском языке. В систематический обзор были включены и проанализированы 15 полнотекстовых статей.

Результаты. Скорость перемещения зубов по данным систематического обзора увеличивается при кортикотомии в 2 раза, при пьезокортикотомии в 1,5 раза, что согласуется с данными других исследований. При этом эффективность перемещения сохраняется в течение не более 2 месяцев после проведения вмешательства.

Выводы. Процедура кортикотомии сокращает время лечения по сравнению с традиционными методами на $30-$ 50\% (6-8 месяцев). Исследуемые методики не оказывали существенного влияния на глубину зондирования, уровень прикрепления, на плотность костной ткани, ротации и наклон перемещаемых зубов. Так как за 2-4 месяца наблюдений никаких осложнений не было выявлено, требуется продолжение исследований для оценки отдаленных результатов в долгосрочной перспективе.
\end{abstract}

Ключевые слова: кортикотомия, пьезокортикотомия, лазеротерапия, ретракция клыков.

Статья поступила: 19.12.2020; исправлена: 14.02.2021; принята: 18.02.2021.

Конфликт интересов: Авторы декларируют отсутствие конфликта интересов.

Для цитирования: Хабадзе 3.С., Балашова М.Е. Эффективность хирургических и нехирургических методов в ортодонтической практике при закрытии постэкстракционных пространств: систематический обзор. Эндодонтия today. 2021; 19(1):31-38. DOI: 10.36377/1683-2981-2021-19-1-31-38.

\title{
The effectiveness of surgical and non-surgical techniques in orthodontic practice in closing post-extraction spaces: a systematic review
}

(C) Z.S. Khabadze', M.E. Balashova²

"'Peoples' Friendship University of Russia" (RUDN University), Moscow, Russia

Abstract:

${ }^{2}$ National Medical Research Center of Dentistry and Oral and Maxillofacial Surgery, Moscow, Russia

Reducing the orthodontic treatment duration is an important issue, especially for adult patients who want to complete treatment faster due to social or aesthetic reasons. In addition, an increase in the duration of orthodontic treatment 
has a number of side effects, therefore, orthodontists and patients are interested in the use of techniques that can accelerate the movement of teeth. Shorter treatment times can be achieved through a combination of orthodontic and surgical or non-surgical techniques.

Aim. To systematically evaluate scientific data on the effectiveness and side effects of surgical and non-surgical methods in accelerating orthodontic treatment in situations with the removal of premolars and subsequent closure of the postextraction spaces.

Materials and methods. Electronic search of articles was carried out using search engines and databases: Cyberleninka, eLIBRARY, Google Scholar, Pub Med, SCOPUS. The publication date criterion was selected from January 2010 to January 2021. Articles included in the systematic review had to meet the following inclusion criteria: controlled clinical trials and randomized controlled clinical trials conducted in humans and published in the last 11 years in English language. The systematic review included and analyzed 15 full-text articles.

Result. According to the systematic review, the speed of movement of teeth increases 2 times with corticotomy, and 1.5 times with piezocorticotomy, which is consistent with the data of other studies. At the same time, the effectiveness of the movement remains for no more than 2 months after the intervention.

Conclusions. The corticotomy procedure reduces the treatment time in comparison with traditional methods by $30-$ $50 \%$ (6-8 months). The investigated techniques did not have a significant effect on the depth of probing, the level of attachment, on the density of bone tissue, rotation and inclination of the moved teeth. Since no complications were identified for 2-4 months of observation, further studies are required to assess long-term results in the long term.

Keywords: corticotomy, piezocorticotomy, laser therapy, canine retraction.

Received: 19.12.2020; revised: 14.02.2021; accepted: 18.02.2021.

Conflict of interests: The authors declare no conflict of interests.

For citation: Z.S. Khabadze, M.E. Balashova. The effectiveness of surgical and non-surgical techniques in orthodontic practice in closing post-extraction spaces: a systematic review. Endodontics today. 2021; 19(1):31-38. DOI: 10.36377/1683-2981-2021-19-1-31-38.

\section{ВВЕДЕНИЕ}

Целью ортодонтического лечения является достижение функционального и эстетического равновесия, что в свою очередь улучшает социальную адаптацию пациентов и повышает качество жизни. Комплексное ортодонтическое лечение в среднем длится от полутора до двух лет. Продолжительность зависит от различных факторов и в большей степени определяется сложностью аномалии прикуса.

Длительный срок лечения является одной из основных причин, по которым пациенты могут отказаться от ортодонтического вмешательства. Сокращение продолжительности ортодонтического лечения является важным вопросом, особенно для взрослых пациентов, которые хотят быстрее завершить лечение в виду социальных или эстетических обстоятельств. Кроме того, увеличение продолжительности ортодонтического лечения имеет ряд побочных эффектов, таких как появление болей или дискомфорта, развитие кариеса, рецессий десны, резорбций корня и др. Поэтому и ортодонты, и пациенты заинтересованы в применении методик, способных ускорить перемещение зубов. Сокращение сроков лечения может быть достигнуто путем сочетания ортодонтического и хирургических или нехирургических методов.

\section{АКТУАЛЬНОСТЬ}

По данным Всемирной Организации Здравоохранения, дистальный прикус является самой распространенной аномалией зубного ряда. Одним из наиболее распространенных методом коррекции зубоальвеолярной формы данного прикуса является лечение с удалением зубов, чаще всего первых или вторых премоляров с последующей ретракцией фронтальной группы зубов и закрытием постэкстракционых пространств. Фаза ретракции клыков обычно длится от 6 до 8 месяцев [1,2], что значительно увеличивает сроки ортодонтического лечения. Для ускорения движения зубов используются различные хирургические и не- хирургические методы: остеотомия, кортикотомия, микро-остеоперфорации, пьезохирургия, лазеротерапия. Для пациентов с дистальным прикусом в сочетании с протрузией и скученностью зубов ортодонтическое лечение с малоинвазивной хирургией занимает промежуточное положение между традиционным лечением и ортогнатической хирургией, при этом сокращает время лечения и снижает риск развития рецидивов.

В последние годы были представлены многочисленные обзорные статьи и тематические исследования, в которых были описаны методики ускорения перемещения зубов. В отечественной и зарубежной литературе до настоящего времени нет четкого представления об этапности ведения таких пациентов, особенностях, на которые необходимо обращать внимание, и использовании дополнительных методов обследования и лечения, необходимых для таких пациентов. Стоит отметить, что в отечественной литературе отсутствуют систематизированные данные по исследуемой проблеме.

Поскольку данные методики являются относительно новыми и могут нести дополнительные риски по сравнению со стандартным лечением необходимо провести систематический обзор, чтобы определить, есть ли достаточно научных данных, подтверждающих использование данных методов и обосновывающих их практическую значимость.

Цель исследования - систематическая оценка научных данных об эффективности и побочных эффектах хирургических и нехирургических методов в ускорении ортодонтического лечения в ситуациях с удалением премоляров и последующим закрытием постэкстракционных пространств.

\section{МАТЕРИАЛ И МЕТОДЫ}

Электронный поиск статей проводился с помощью поисковых систем и баз данных: Cyberleninka, eLIBRARY, Google Scholar, Pub Med, SCOPUS. Элек- 
Таблица 1. Критерии включения и исключения

Table 1. Inclusion and exclusion criteria

\begin{tabular}{|l|l|}
\hline Критерии включения & Критерии исключения \\
\hline $\begin{array}{l}\text { 1. Рандомизированные контролируемые клинические исследование, по типу } \\
\text { "split-mouth design" }\end{array}$ & $\begin{array}{l}\text { 1. Нерандомизированные неконтролируемые исследования, наблюдательные } \\
\text { исследования, описательные исследования }\end{array}$ \\
\hline 2. Здоровые пациенты, нуждающиеся в ортодонтическом лечении & 2. Лабораторные, неклинические исследования, исследования на животных \\
\hline $\begin{array}{l}\text { 3. Ортодонтическое лечение в сочетании с кортикотомией, пьезохирургией и } \\
\text { лазеротерапией в сравнении со стандартным ортодонтическим лечением }\end{array}$ & 3. Систематические обзоры, мета-анализы, тезисы, мнения исследователей \\
\hline 4. Ретракция клыков & $\begin{array}{l}\text { 4. Вмешательства, не связанные с дистракционным остеогенезом, Остеото- } \\
\text { мией, кортикотомией }\end{array}$ \\
\hline 5. Скорость перемещения зубов и продолжительность лечения & $\begin{array}{l}\text { 5. Системные заболевания, приём лекарственных препаратов, заболевания } \\
\text { органов полости рта, плохая гигиена }\end{array}$ \\
\hline & 6. Неанглоязычные публикации \\
\hline
\end{tabular}

тронный поиск проводился по следующим ключевым словам: corticotomy, orthodontics, accelerated tooth movement, minimally invasive, surgical, canine retraction. Рассматривались статьи на русском и английском языках. В виду отсутствия публикаций в отечественной литературе в систематический обзор были включены статьи только на английском языке.

Критерий даты публикации статей был выбран с января 2010 г. по январь 2021 г. Статьи, включенные в этот систематический обзор, должны были соответствовать следующим критериям включения: контролируемые клинические испытания и рандомизированные контролируемые клинические испытания, проведенные на людях и опубликованные в течение последних 11 лет на английском языке. В статьях должна содержаться подробная информация о результатах и параметрах лечения. Были включены клинические исследования, в которых проводилось удаление премоляров с последующим закрытием постэкстракционного пространства без и с использованием средств для усиления анкоража. Выводы должны были быть научно обоснованными. Были исключены клинические испытания, которые давали недостаточную информацию, а также исследования, проведенные на животных.

Статьи изучали по названию и цели, затем по резюме, далее был проведен полнотекстовый анализ, включая материалы и методы. Интерес представляли исследования, отражающие клиническое обоснование применения различных методик для ускорения перемещения зубов в механике закрытия постэкстракционных пространств. Полученные исследования должны были представлять первичные результаты о скорости перемещения зубов, сроках лечения. Вторичными результатами была информация об осложнениях и других эффектах, возникающих в процессе лечения.

После оценки найденной информации и отбора статей в соответствии с критериями включения был проведен заключительный анализ отдельных исследований. Процесс выборки и анализа исследований представлен в виде блок-схемы (рисунок 1).

\section{РЕЗУЛЬТАТЫ}

В результате электронного поиска было найдено 157 исследований по ключевым словам и резюме. Дупликаты исследований были исключены, названия и тезисы 67 статей были проверены на соответствие критериям отбора. Статьи, которые не соответствовали критериям, были исключены. Подробная инфор- мация о количестве работ и причинах исключения на каждом этапе представлена на рис. 1. В итоге в систематический обзор были включены и проанализированы 15 полнотекстовых статей.

Характеристики 15 отобранных исследований представлены в таблице 2. Все публикации являются рандомизированными клиническими исследованиями по типу "split-mouth design", которые проводились на людях, за исключение исследования с виртуальным моделированием [3].

Таблица 2. Характеристика исследований, включенных в систематический обзор.

Характеристики участников: общее количество пациентов составило 242 человека (136 женщин и 50 мужчин) разной этнической принадлежности, которым проводилось лечение на лигатурной металлической брекет-системе. В двух исследованиях пол пациентов не указывался [4, 5]. Средний возраст 20-21 год. План лечения включал в себя двустороннее удаление первых постоянных премоляров на верхней и нижней [4] челюсти. Период наблюдения в среднем составил 2 - 4 месяца.

В 9 исследованиях проводилась кортикотомия, в 2 пьезокортикомтомия, в 1 - лазеротерапия, в 3 были выполнены сочетанные вмешательства. Почти во всех рандомизированных клинических испытаниях, вмешательства проводились с вестибулярной стороны альвеолярного отростка, за исключением некоторых статей, где процедура была двусторонней [4, 6, 7, 8]. Для закрытия постэкстракционных пространств в большинстве случаев использовались никель-титановые закрывающие пружины с нагрузкой в 150 [3,4,6,7,8,9,10,11,12,13,14,15] и 250 [16] грамм. Реже для закрытие использовался эластический чейн [4,9,15], tie-back [4] и специальный дистрактор с винтом Hyrex [17].

\section{ОБСУЖДЕНИЕ}

\section{Скорость ретракции}

Скорость проведения процедуры ретракции клыков по общепринятым методикам составляет 0,5 - 1 мм в месяц, что занимает от 5 до 9 месяцев лечения. Длительное лечение увеличивает риск развития кариеса, резорбции корней, проблем с пародонтом, также снижает кооперацию с пациентами. Многие исследования доказывают, что при перемещении зубов в волокнистую костную ткань, только что созданную дистракционным остеогенезом, перемещение зуба может быть 1,2 мм в неделю [16].

Результаты всех исследований подтверждают, что хирургические и нехирургические методы способны увеличить скорость перемещения зубов. Вмеша- 


\title{
34 06зоры / Reviews
}

тельства направлены на активирование феномена региональной акселерации (Regional Acceleratory Phenomenon) по теории Фроста [17]. Скорость перемещения зубов по данным систематического обзора увеличивается при кортикотомии в 2 раза, при пьезокортикотомии в 1,5 раза, что согласуется с данными других исследований $[11,12,13,18]$. При этом эффективность перемещения сохраняется в течение не более 2 месяцев после проведения вмешательства. Это подтверждает, что феномен региональной акселерации является транзиторной стадией ремоделирования костной ткани $[19,20]$. Fischer также считал, что кортикотомия способствует более быстрому движению зубов. Процедура уменьшает плотность костной ткани, усиливает кровообращение в области вмешательства, в последующем ускоряет остеогенные процессы. Все изменения пропорциональны степени повреждения тканей [21].

Al-Naoum et al. [6] сообщили, что скорость движения зубов была в 4 раза выше на стороне кортикотомии в течение первых трех дней и в 3 раза выше с пятого дня.
Это изменение скорости движения согласуется с результатами других авторов. Aboul-Ela et al. [13] определили, что скорость движения зубов в два 2 выше в течение первых двух месяцев на стороне кортикотомии.

Результаты исследования Alfawal et al. [12] показали, что методы пьезокортикотомии и лазерной абляции эффективны для ускорения ретракции клыков. Можно утверждать, что кортикотомия является несколько агрессивным по сравнению с так называемой процедурой "кортицизации", без сепарации лоскута. Однако, сокращая продолжительность хирургической операции, данные методы не обеспечивают хирургу широкого и четкого доступа, который обычно получается после откидывания лоскутов при кортикотомии.

Наиболее перспективными хирургическими методами лечения являются наименее инвазивные, такие как микро-остеоперфорации [22]. Исследование Agrawal et al. [23] доказало эффективность микроостеоперфораций ортодонтическими минивинтами в ускорении перемещении зубов. Однако при кортикотомии ретракция быстрее на 0,5-1мм в месяц, чем

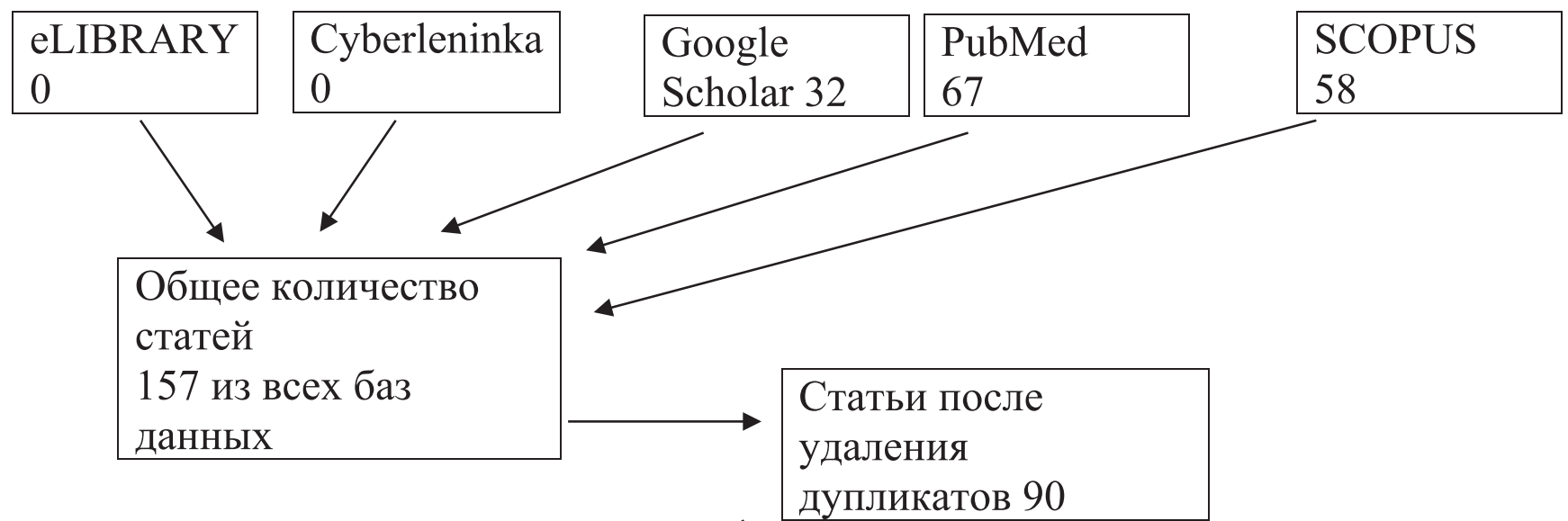

Статьи отобранные по названию и резюме 67

\begin{tabular}{|l|}
\hline Исключены не \\
полнотекстовые \\
исследования \\
\hline
\end{tabular}

\section{5 полнотекстовых исследования}

\begin{tabular}{|l|}
\hline Исключены статьи, не \\
соответствующие \\
критериям включения \\
\hline
\end{tabular}

\author{
15 полнотекстовых \\ исследования
}

Рис. 1. Блок-схема, отражающая процесс выбора исследований

Fig. 1. Block-scheme demonstrating the study selection process 
Таблица 2. Характеристика исследований, включенных в систематический обзор. Table 2. Description of the studies included in the systematic review.

\begin{tabular}{|c|c|c|c|c|c|}
\hline Автор, год & Пациенты & $\begin{array}{c}\text { Тип Вмешательства, способ } \\
\text { ретракции }\end{array}$ & $\begin{array}{l}\text { Методы } \\
\text { оценки }\end{array}$ & Результат & $\begin{array}{c}\text { Неблагоприятные по- } \\
\text { следствия/ вторичные } \\
\text { результаты }\end{array}$ \\
\hline $\begin{array}{l}\text { 1. Al-Naoum et al., } \\
2014\end{array}$ & $\mid \begin{array}{l}30 \text { (15 мужчин, } 15 \\
\text { женщин), возраст } \\
15-24,2 \text { класс } 1 \text { и } \\
2 \text { подкласс Энгля }\end{array}$ & $\begin{array}{l}\text { Двустроронняя кортикотомия в об- } \\
\text { ласти клыков и первых премоляров } \\
\text { через } 4 \text { недели после удаления. } \\
\text { Дуга } 0.019 \text { * } 0.025 \text { SS, NiTi пружи- } \\
\text { на } 150 \text { гр, небный бюгель }\end{array}$ & $\begin{array}{l}\text { Внутриротовые } \\
\text { измерения } \\
\text { штангельцирку- } \\
\text { лем, гипсовые } \\
\text { модели, анкети- } \\
\text { рование }\end{array}$ & $\begin{array}{l}\text { Результат не зависит от пола. } \\
\text { Увеличение перемещения в } \\
\text { 2-4 раза при кортикотомии, } \\
\text { при процедуре наблюдались } \\
\text { умеренная боль и дискомфорт }\end{array}$ & $\begin{array}{l}\text { Использование небного бюгеля } \\
\text { усиливает анкораж моляров }\end{array}$ \\
\hline $\begin{array}{l}\text { 2. Krishnan, et al. } \\
2013\end{array}$ & возраст 18-25 & $\begin{array}{l}\text { Двустроронняя кортикотомия в об- } \\
\text { ласти клыков и первых премоляров } \\
\text { одновременно с удалением. Дуга } \\
0.019 \text { * } 0.025 \text { SS, NiTi пружина } 150 \\
\text { гр, чейн, tie-back }\end{array}$ & $\begin{array}{l}\text { Внутриротовые } \\
\text { измерения } \\
\text { штангельцирку- } \\
\text { лем, гипсовые } \\
\text { модели, анкети- } \\
\text { рование }\end{array}$ & $\begin{array}{l}\text { Средняя скорость ретракции } \\
\text { на верхней челюсти (5,89 мм) } \\
\text { больше, чем на нижней (5,75 } \\
\text { мм) за } 9 \text { недель. На верхней } \\
\text { челюсти потеря анкоража } \\
\text { больше. }\end{array}$ & $\begin{array}{l}\text { Потеря анкоража больше при } \\
\text { использовании чейна. Скорость } \\
\text { перемещения зависит от } \\
\text { плотности костной ткани и } \\
\text { жевательной нагрузки }\end{array}$ \\
\hline $\begin{array}{l}\text { 3. Bhattacharya et } \\
\text { al. } 2014\end{array}$ & \begin{tabular}{|l|}
20 пациентов \\
(2 мужчин и 18 \\
женщин), возраст \\
$15-25$ \\
\end{tabular} & $\begin{array}{l}\text { Двустроронняя кортикотомия } \\
\text { фронтального сегмента верхней } \\
\text { челюсти одновременно с удале- } \\
\text { нием. Дуга } 0.016 \text { * } 0.22 \text { SS. NiTi } \\
\text { пружина } 250 \text { гр, небный бюгель }\end{array}$ & $\begin{array}{l}\text { Компьютерная } \\
\text { томография }\end{array}$ & $\begin{array}{l}\text { Сокращение сроков лечения } \\
2,5 \text { месяца при кортикотомии }\end{array}$ & $\begin{array}{l}\text { Увеличение толщины кости за } \\
\text { счет добавления остеоиндук- } \\
\text { тивного материала. Наиболь- } \\
\text { ший прирост костной ткани у } \\
\text { альвеолярного гребня } \\
\end{array}$ \\
\hline 4. Yang et al. 2015 & \begin{tabular}{|l|} 
Смоделирован- \\
ные КТ снимки \\
верхней челюсти, \\
24 геометрических \\
модели зубных \\
рядов
\end{tabular} & $\begin{array}{l}20 \text { модификаций разрезов при } \\
\text { кортикотомии, сегментированные } \\
\text { квадратные дуги, смоделирован- } \\
\text { ная нагрузка } 100 \text { гр }\end{array}$ & $\begin{array}{l}\text { 3D конечно- } \\
\text { элементный } \\
\text { анализ }\end{array}$ & $\begin{array}{l}\text { Перемещение активнее в } \\
\text { моделях с дистальными раз- } \\
\text { резами и их комбинациями. } \\
\text { Чем дальше разрез от клыка, } \\
\text { тем меньше перемещение. } \\
\text { Ширина разреза не оказывает } \\
\text { значительного влияния на } \\
\text { перемещение. }\end{array}$ & $\begin{array}{l}\text { Увеличение расстояния и } \\
\text { ширины разреза уменьшает } \\
\text { напряжение в периодонте. } \\
\text { Разрез более } 5 \text { мм от клыка } \\
\text { не ускоряет перемещение. } \\
\text { Непрерывный циркулярный } \\
\text { разрез увеличивает напряжение } \\
\text { в губчатой кости. Дистальные } \\
\text { разрезы и их комбинации } \\
\text { снижают нагрузку на периодонт } \\
\text { и внешнюю резорбцию корня. }\end{array}$ \\
\hline $\begin{array}{l}\text { 5. Agrawal et al., } \\
2018\end{array}$ & $\begin{array}{l}10 \text { пациентов } \\
(8 \text { женщин, } 2 \\
\text { мужчин), возраст } \\
18-25 \text { лет }\end{array}$ & $\begin{array}{l}\text { Кортикотомия и микро-остеопер- } \\
\text { форации ортодонтическими мини- } \\
\text { винтами с вестибулярной стороны. } \\
\text { Ретракция через } 2 \text { недели после } \\
\text { операции. }\end{array}$ & КЛКТ & $\begin{array}{l}\text { Оба метода увеличивают } \\
\text { скорость ретракции клыков за } \\
\text { короткий промежуток времени. } \\
\text { При кортикотомии ретракция } \\
\text { быстрее на 0,5-1 месяц. }\end{array}$ & $\begin{array}{l}\text { Увеличение толщины кости } \\
\text { за счет добавления костного } \\
\text { материала. Микро-остеоперфо- } \\
\text { рации менее травматичны для } \\
\text { пародонта. Резорбция корня } \\
\text { клыка статистически не значима }\end{array}$ \\
\hline $\begin{array}{l}\text { 6. Kumar } \mathrm{N} \text { et al., } \\
2013\end{array}$ & \begin{tabular}{|l|}
7 пациентов \\
(5 женщин и 2 \\
мужчин), средний \\
возраст 18 лет \\
\\
\\
\end{tabular} & $\begin{array}{l}\text { Кортикотомия с вестибуляр- } \\
\text { ной стороны одновременно с } \\
\text { удалением. Остеотомия костного } \\
\text { блока в области клыка, перфора- } \\
\text { ции межсептальной перегородки } \\
\text { и интраальвеолярные насечки. } \\
\text { Дистрактор с винтом Нугех. После } \\
\text { дистракции- брекет-система. }\end{array}$ & $\begin{array}{l}\text { Гипсовые } \\
\text { модели, фото- } \\
\text { графии, цефа- } \\
\text { лометрическая, } \\
\text { панорамная и } \\
\text { периапикальная } \\
\text { рентгеногра- } \\
\text { фия, ЭоД } \\
\end{array}$ & $\begin{array}{l}\text { Корпусная ретракция верхних } \\
\text { и нижних клыков на 6,5 мм } \\
\text { за 2-3 недели. Сокращение } \\
\text { срока лечения на 6-9 месяцев. } \\
\text { Остеотомия и дистракция- } \\
\text { метод выбора для ретракции } \\
\text { анкилоризованных зубов }\end{array}$ & $\begin{array}{l}\text { Ретракция клыков за } 16 \text { дней } \\
\text { уменьшает риск резорбции } \\
\text { корней. Незначительная потеря } \\
\text { анкоража (0-2мм), больше на } \\
\text { верхней челюсти. Отсутствие } \\
\text { перегрузки пародонта и нега- } \\
\text { тивного влияния на пульпу. }\end{array}$ \\
\hline $\begin{array}{l}\text { 7. Hatrom A. A. et. } \\
\text { al., } 2020\end{array}$ & \begin{tabular}{|l|}
26 пациентов, во3- \\
раст $16-26$ лет 2 \\
класс 1 подкласс \\
\end{tabular} & $\begin{array}{l}\text { Пьезокортикотомии с вестибуляр- } \\
\text { ной стороны и в области лунки } \\
\text { удаленного премоляра. Дуга } 0.018 \\
\text { x } 0.22 \text { SS. NiTi пружина } 250 \text { гр, ми- } \\
\text { нивинты между первым моляром и } \\
\text { вторым премоляром. }\end{array}$ & $\begin{array}{l}\text { Компьютерная } \\
\text { томография, } \\
\text { фотографии, } \\
\text { цифровые } \\
\text { модели. }\end{array}$ & $\begin{array}{l}\text { Пьезокортикотомия ускоряет } \\
\text { ретракцию клыков в } 2 \text { раза }\end{array}$ & $\begin{array}{l}\text { Минивинты уменьшают потерю } \\
\text { анкоража. Резорбция корня } \\
\text { правого и левого централь- } \\
\text { ного резца и правого клыка и } \\
\text { болевые ощущения меньше при } \\
\text { пьезохирургии }\end{array}$ \\
\hline $\begin{array}{l}\text { 8. Mahmoudzadeh } \\
\text { et al., } 2020\end{array}$ & $\begin{array}{l}12 \text { пациентов } \\
\text { (9 женщин и } 3 \\
\text { мужчин), возраст } \\
15-30 \text { лет }\end{array}$ & $\begin{array}{l}\text { Абляция Er,Cr:YSGG-лазером } \\
\text { десны мезиально и дистально от } \\
\text { клыка на } 2 \text { мм ниже альвеоляр- } \\
\text { ного гребня до мукогингивальной } \\
\text { границы, глубиной 2-3 мм. Дуга } \\
0.016^{\star} 0.022 \text { SS, пружина } 150 \text { гр, } \\
\text { чейн }\end{array}$ & $\begin{array}{l}\text { КТ, цифровые } \\
\text { модели, анкети- } \\
\text { рование }\end{array}$ & $\begin{array}{l}\text { Скорость ретракции клыков } \\
\text { через } 1 \text { месяц была больше в } \\
2,5 \text { раза на стороне лазерной } \\
\text { абляции. }\end{array}$ & $\begin{array}{l}\text { Лазе оказывает бактерицидный } \\
\text { эффект. Отсутствие боли, } \\
\text { отека, меньше наклон коронки } \\
\text { клыка при абляции. Отсутствие } \\
\text { потери анкоража и прикреплен- } \\
\text { ной десны }\end{array}$ \\
\hline $\begin{array}{l}\text { 9. Omidkhoda et } \\
\text { al., } 2020\end{array}$ & $\begin{array}{l}17 \text { пациентов } \\
\text { (7 мужчин и } 10 \\
\text { женщин, возраст } \\
14-30 \text { лет }\end{array}$ & $\begin{array}{l}\text { Ретракция через } 3 \text { месяца после } \\
\text { удаления. Перфорации пьезото- } \\
\text { мом дистально и мезиально от } \\
\text { клыка с двух сторон. NiTi пружина } \\
150 \text { гр }\end{array}$ & $\begin{array}{l}\text { Фотографии, } \\
\text { модели }\end{array}$ & $\begin{array}{l}\text { Перфорации пьезотомом } \\
\text { не увеличивают скорость } \\
\text { ретракции }\end{array}$ & $\begin{array}{l}\text { Ротация клыков, потеря анко- } \\
\text { ража были статистически не } \\
\text { значимыми }\end{array}$ \\
\hline
\end{tabular}




\begin{tabular}{|c|c|c|c|c|c|}
\hline Автор, год & Пациенты & $\begin{array}{c}\text { Тип Вмешательства, способ } \\
\text { ретракции }\end{array}$ & $\begin{array}{l}\text { Методы } \\
\text { оценки }\end{array}$ & Результат & $\begin{array}{c}\text { Неблагоприятные по- } \\
\text { следствия/ вторичные } \\
\text { результаты } \\
\end{array}$ \\
\hline $\begin{array}{l}\text { 10.Abbas et. al., } \\
2016\end{array}$ & $\begin{array}{l}20 \text { пациентов, в03- } \\
\text { раст } 15-25 \text { лет }\end{array}$ & $\begin{array}{l}\text { Ретракция клыков после удаления, } \\
\text { кортикотомия и пьезохирургия } \\
\text { с вестибулярной стороны. Дуга } \\
0.016^{\star} 0.022 \text { SS. NiTi пружина } \\
150 \text { гр }\end{array}$ & $\begin{array}{l}\text { Модели, } \\
\text { фотографии } \\
\text { моделей, } \\
\text { компьютерная } \\
\text { томография }\end{array}$ & $\begin{array}{l}\text { При кортикотомии скорость } \\
\text { перемещения увеличивается } \\
\text { в } 2 \text { раза, при пьезохирургии в } \\
1,5 \text { раза. }\end{array}$ & $\begin{array}{l}\text { Резорбция корня больше в } \\
\text { группах без вмешательств. } \\
\text { Нет различий в наклоне, } \\
\text { инклинации и ротации клыков, } \\
\text { движении моляров, резорбции } \\
\text { корней, изменений в пародонте } \\
\text { при кортикотомии и пьезохи- } \\
\text { рургии }\end{array}$ \\
\hline $\begin{array}{l}\text { 11.Alfawal et al., } \\
2018\end{array}$ & $\begin{array}{l}36 \text { пациентов (12 } \\
\text { мужчин, } 24 \text { жен- } \\
\text { щины), возраст } \\
15-27 \text { лет }\end{array}$ & $\begin{array}{l}\text { Ретракция после удаления пре- } \\
\text { моляров. Пьезохирургия и абляция } \\
\text { лазером Er:YAG c вестибулярной } \\
\text { стороны. Дуга } 0.019 \text { × } 0.025 \text { SS, } \\
\text { NiTi пружина } 150 \text { гр, небный } \\
\text { бюгель }\end{array}$ & $\begin{array}{l}\text { Гипсовые } \\
\text { модели, от- } \\
\text { цифрованные } \\
\text { фотографии } \\
\text { моделей. }\end{array}$ & $\begin{array}{l}\text { Активное перемещение в } \\
\text { первые } 2 \text { месяца. Разница в } \\
\text { скорости между группами не- } \\
\text { значительная. Методы можно } \\
\text { использовать как дополни- } \\
\text { тельные }\end{array}$ & $\begin{array}{l}\text { Не было выявлено достоверных } \\
\text { различий между группами в по- } \\
\text { тере анкоража, ротации клыков }\end{array}$ \\
\hline $\begin{array}{l}\text { 12.Aboul-Ela et } \\
\text { al., } 2011\end{array}$ & $\begin{array}{c}13 \text { пациентов } \\
\text { (5 мужчин и } 8 \\
\text { женщин), средний } \\
\text { возраст } 19 \text { лет }\end{array}$ & $\begin{array}{c}\text { Кортикотомия с вестибуляр- } \\
\text { ной стороны одновременно с } \\
\text { удалением. Дуга } 0.018^{\star} 0.22 \mathrm{SS} . \\
\text { NiTi пружина } 150 \text { гр, минивинты } \\
\text { между первым моляром и вторым } \\
\text { премоляром. }\end{array}$ & $\begin{array}{l}\text { Гипсовые } \\
\text { Модели, индекс } \\
\text { Silness и Loe }\end{array}$ & $\begin{array}{c}\text { В первые } 2 \text { месяца наибольшая } \\
\text { скорость перемещения после } \\
\text { кортикотомии. у } 45 \% \text { паци- } \\
\text { ентов отношения клыков по I } \\
\text { классу были достигнуты через } \\
\text { 2-3 месяца. На контрольной } \\
\text { стороне за } 4 \text { месяца не было } \\
\text { достигнуто } 1 \text { класса по клыкам. }\end{array}$ & $\begin{array}{c}\text { Нет статистически значимой } \\
\text { разницы в индексах зубного } \\
\text { налета, потери прикрепления, } \\
\text { рецессии десны и глубины зон- } \\
\text { дирования между группами до и } \\
\text { после операции. Длительность } \\
\text { феномена региональной аксе- } \\
\text { лирации повышает десневой } \\
\text { индекс Loe Silness. }\end{array}$ \\
\hline $\begin{array}{l}\text { 13.Jahanbakhshi, } \\
\text { et al., } 2016\end{array}$ & 15 женщин, 25 лет & $\begin{array}{c}\text { Ретракция одновременно с удале- } \\
\text { нием. Кортикотомия с вестибуляр- } \\
\text { ной стороны. Дуга } 0.016^{\star} 0.016 \mathrm{SS} \\
\text { с закрывающими петлями, мини- } \\
\text { винты между первым моляром и } \\
\text { вторым премоляром. }\end{array}$ & $\begin{array}{c}\text { Внутриротовые } \\
\text { измерения } \\
\text { штангельцир- } \\
\text { кулем }\end{array}$ & $\begin{array}{c}\text { Кортикотомия увеличивает } \\
\text { скорость ретракции в } 2 \text { раза в } \\
\text { первые } 2 \text { месяца, в 1,5 раза на } \\
3 \text { месяце. }\end{array}$ & $\begin{array}{c}\text { Потери анкоража не было ни в } \\
\text { одной из групп }\end{array}$ \\
\hline $\begin{array}{l}\text { 14. Farid et.al. } \\
2019\end{array}$ & $\begin{array}{c}16 \text { женщин, } 17-25 \\
\text { лет }\end{array}$ & $\begin{array}{c}\text { Двустороння кортикотомия от } \\
\text { клыка до второго премоляра, дву- } \\
\text { сторонняя низкоинтенсивная ла- } \\
\text { зерная терапия в день операции. } \\
\text { Дуга } 0.017^{\star} 0.22 \text { SS. NiTi пружина } \\
150 \text { гр, небный бюгель }\end{array}$ & $\begin{array}{c}\text { Гипсовые моде- } \\
\text { ли, трехмерные } \\
\text { цифровые } \\
\text { модели }\end{array}$ & $\begin{array}{c}\text { Сочетание лазера и кортикото- } \\
\text { мии не эффективно. Средняя } \\
\text { скорость ретракции отличается } \\
\text { на } 0,7 \text { мм/месяц. В } 1 \text { месяц } \\
\text { скорость меньше на стороне } \\
\text { кортикотомии и лазеротера- } \\
\text { пии, на } 2 \text { и } 3 \text { месяце статисти- } \\
\text { чески значимой разницы нет }\end{array}$ & $\begin{array}{c}\text { Потеря акоража статистически } \\
\text { незначимая }\end{array}$ \\
\hline $\begin{array}{l}\text { 15. Baeshen, H.A., } \\
2020\end{array}$ & $\begin{array}{l}20 \text { пациентов (2 } \\
\text { мужчин } 16 \text { жен- } \\
\text { щин) 13-21 год, }\end{array}$ & $\begin{array}{c}\text { Частичная кортикотомия с } \\
\text { вестибулярной стороны, ретракция } \\
\text { через } 2 \text { недели после удаления. } \\
\text { Дуга } 0.018 \text { SS, чейн } 150 \text { гр, неб- } \\
\text { ный бюгель }\end{array}$ & $\begin{array}{c}\text { Панорамная и } \\
\text { периапикальная } \\
\text { рентгенография } \\
\text { гипсовые моде- } \\
\text { ли, цифровые } \\
\text { фото }\end{array}$ & $\begin{array}{c}\text { Кортикотомия увеличивает } \\
\text { скорость ретракции на 0,15 } \\
-0,2 \text { мм/неделю }\end{array}$ & $\begin{array}{c}\text { Нет статистически значимой } \\
\text { разницы в потере анкоража, } \\
\text { резорбции корня, ротации } \\
\text { клыков, глубине зондирования, } \\
\text { плотности кости. Использова- } \\
\text { ние чейна уменьшает потерю } \\
\text { анкоража }\end{array}$ \\
\hline
\end{tabular}

при микро-остеоперфорациях. Вторая методика как менее травматичная подходит для пациентов с сопутствующими заболеваниями, пародонтопатологиями, заболеваниями слизистой оболочки полости рта.

В исследованиях Abbas et. al. [11] и Hatrom A. A. et. al [15] было доказано, что проведение пьезокортикотомии с вестибулярной стороны и в области альвеолы удаленного премоляра увеличивает скорость закрытия пространства в 2 раза по сравнению со стандартной методикой. При этом методе лучше использовать более сильную никель-титановую пружину 250 гр, а не 150 гр как при кортикотомии. Использование пьезохирургии улучшает заживление, не вызывая остеонекротических повреждений, обеспечивает сохранение целостности корня, не раздражает мягкие ткани и не нарушает их кровоснабжение [11], что является актуальным методов для пациентов с сопутствующей патологией.

Для ретракции в клинических ситуациях с анкилозированными зубами, дилацерацией корня, длинными корнями, при макродентии, пародонтопатологиями методом выбора является кортикотомия и остеотомия с использованием дистрактора с винтом Hyrex [16]. В исследовании ретракция клыков была достигнута через 12-16 дней, что является самым минимальным периодом. Мезиальное смещение моляров или резорбция корней не успевают произойти за такой короткий срок. Использование Er,Cr:YSGG-лазера также подходит для применения у пациентов с заболеваниями пародонта [9], так как метод менее инвазивный, а послеоперационные осложнения, потеря анкоража и прикрепленной десны в исследовании отсутствовали. Авторы сообщали об увеличении скорости ретракции клыков через 1 месяц в 2 раза. Однако стоит помнить о кратковременном эффекте лазеротерапии. Поэтому процедура должна проводится чаще, чем остальные (каждые 2-3 недели).

Еще один менее травматичный метод, модифицированная методика пьезопункции [10], не оказывал существенного влияния на скорость ретракции клыков. 
Поэтому исследования с повторными пьезопункциями через регулярные промежутки времени рекомендуются для изучения преимуществ при ортодонтическом перемещении зубов. При сравнении пьезокортикотомии и лазерной абляции [12] было выявлено, что первый метод ускоряет перемещение зубов на 0,012мм/ месяц больше, чем второй. Выводы согласуются с результатами Aksakalli et al. [24] и Abbas et al. [11], которые сообщают, что пьезокортикотомия способна значительно ускорить скорость ретракции клыков в 1,5-2 раза в течение первых 2-3 месяцев. Согласно данным Farid et.al. [8] использование лазерной абляции coвместно с кортикотомией является не эффективным.

Таким образом, пьезокортикотомия и лазерная абляция являются возможными дополнительными методами для ускорения ортодонтического движения зубов, поскольку они, как было обнаружено, сокращают время ретракции клыков примерно на 25\%. В то время как кортикотомия и микро-остеоперфорации способны ускорить перемещение на 30-40\%.

По данным Krishnan et al. [4] средняя скорость peтракции за 9 недель на верхней челюсти была больше (5,89 мм), чем на нижней (5,75 мм) по причине плотности костной ткани. При это на верхней челюсти происходила большая потеря анкоража - 1,33 мм, чем на нижней- 1,08 мм. Кроме того, при использовании эластофорса 200 гр потеря анкоража была больше, чем при использовании закрывающих пружин и tie-back. Было обнаружено, что причина повышенной ретракции с одной из сторон может быть связана с привычным жеванием на функциональной определенной стороне.

Согласно данным исследования Yang et al. [3] расположение места разреза при кортикотомии также влияет на скорость перемещения зубов. Чтобы ускорить перемещение лучше проводить разрезы не более 4 мм дистально от клыка, дополнительно проводят разрезы с небной и вестибулярной стороны. Непрерывный циркулярный разрез увеличивает напряжение в губчатой кости, а дистальные разрезы и их комбинации снижают нагрузку на пародонт, уменьшая риск развития рецессии десны и резорбции корня.

\section{Побочные эффекты и вторичные результаты Анкораж}

Любая механика в ортодонтии, которая применяется больше трех недель для ретракции клыков, приводит к потере анкоража. Для уменьшения потери анкоража в исследованиях использовались ортодонтические минивинты $[13,15,18]$ и небный бюгель $[6,7,8,12,14$,$] . Зна-$ чительной потери анкоража (более 2 мм) ни в одном из исследований не наблюдалось. При пьезохирургии и лазерной абляции не было выявлено достоверных различий между группами в потере анкоража [12]. Кортикотомия помогает быстрее перемещать зубы и устойчивее фиксировать анкрораж [4].

Уменьшение потери анкоража при использовании эластофорса объясняется тем фактом, что клыки перемещаются довольно быстро (14-20 недель), в то время как моляры все еще находятся в запаздывающей фазе движения [14]. К тому же сила эластичной цепочки может быть недостаточно высокой, чтобы инициировать движение моляров. Потерю анкоража уменьшает лигатурное связывание моляров и второго премоляра, а также перфорация стенок альвеолы после удаления зуба $[6,11,15]$. Leethanakul et al. (5) пришли к выводу, что единственным значимым фактором, коррелирующим с ретракцией клыков, является количество кости, оставшейся в области мезиальной стенки лунки удаленного премоляра[25].

\section{Резорбция корней}

Общепризнано, что лучший способ минимизировать резорбцию корня -это завершить движение зуба за короткое время. Резорбция корня начинается через 2-3 недели после применения ортодонтической силы и продолжается в течение всего времени применения силы. Abbas et al. [11] наблюдали более низкую степень резорбция корня в группе кортикотомии. Однако двумерные ограничения периапикальных рентгенограмм делают эти результаты ненадежными, необходимо проведение компьютерных томограмм. В исследовании Agrawal et al. [23] резорбция корня больше на стороне без вмешательств. Так как резорбция корня начинается через 2-3 недели, использование дистализатора [16] является эффективным методов для перемещения в случаях, если корень уже имеет признаки резорбции.

По данным исследований не было обнаружено статистически значимой разницы в индексах зубного налета, потери прикрепления, рецессии десны и глубины зондирования между группами до и после операции односторонней кортикотомии, пьезокортикотомии.

\section{Боль и дискомфорт}

Согласно исследованию Al-Naoum et al. [6] пациенты выбрали процедуру кортикотомии, как менее болезненную по сравнению с удалением премоляров. Выраженная боль и дискомфорт наблюдались в первые сутки после кортикотомии. у 66,66\% пациентов боль практически прошла через неделю. У $80 \%$ пациентов наблюдался средний отек, который был максимальным на третий день. В некоторых исследованиях авторы заявили, что процедура альвеолярной кортикотомии оказалась хорошо переносимой пациентами с умеренной степенью боли и дискомфорта [6, 9].

\section{Костная пластинка}

Сочетание костной пластики с кортикотомией увеличивает объем костной ткани, что приводит к значительно большему прикреплению десны и способствует восстановлению альвеолярных кортикальных костных фенестраций и дигесценций [11]. Трансплантация увеличивает объем кости для перемещаемого зуба, что позволяет ему занять более стабильное положение. Bhattacharya et al. [7] доказали, что при кортикотомии можно увеличить объём костной, используя остеопластические материалы. При этом наибольший прирост костной ткани наблюдался в области альвеолярного гребня.

\section{выводы}

Процедура кортикотомии сокращает время лечения по сравнению с традиционными методами на 3050\% (6-8 месяцев). Традиционная техника кортикотомии, выполняемая с помощью хирургических боров и наконечников, дает более быстрые результаты, чем пьезохирургия или лазеротерапия. Однако послеоперационный период более благоприятно протекал при использованию двух последних методик. К тому же применение лазерных технологий значительно улучшает заживляющие способности кости. Исследуемые методики не оказывали существенного влияния на глубину зондирования, уровень прикрепления, на плотность костной ткани, ротации и наклон перемещаемых зубов. Так как за 2-4 месяца наблюдений никаких осложнений не было выявлено, требуется продолжение исследований для оценки отдаленных результатов в долгосрочной перспективе. Методики кортикотомии, пьезокортикотомии и лазеротерапии не являются вза- 
имозаменяемым, а зачастую применяются в сочетании в зависимости от клинической ситуации.

Во всех исследованиях феномен ускорения перемещения зубов был обратимым явлением. В связи с этим необходимы дальнейшие исследования, направленные на изучение эффективности повторных вмешательств. Использование перечисленных методик позволяет сократить время лечения и, следовательно, нежелательные эффекты, связанные с длительным лечением.

Клинические ситуации, в которых потеря анкроража выгодна или не критична можно проводить стандарт-

\section{СПИСОК ЛИТЕРАТУРЫ / REFERENCES:}

1. Viwattanatipaa $\mathrm{N}$, Charnchairerk $\mathrm{S}$ The effectiveness of corticotomy and piezocision on canine retraction. Korean $\mathrm{J}$ Orthod 2018;48(3):200-211.

2. Alfawal MH, Hajeer MY, Ajaj MA, Hamadah O, Brad B. Effectiveness of minimally invasive surgical procedures in the acceleration of tooth movement: a systematic review and meta-analysis. Prog Orthod.2016.17(1):33.

3. Yang C, Wang C, Deng F, Fan Y Biomechanical effects of corticotomy approaches on dentoalveolar structures during canine retraction: A 3-dimensional finite element analysis. Am J Orthod Dentofacial Orthop. 2015;148:457-465.

4. Krishnan P, Shetty $S$, Husain A. An adjunctive minor surgica procedure for increased rate of retraction. J Pharm Bioall Sci 2013;5:39 42.

5. N.H. Abbas, N.E. Sabet, I.T. Hassan, Evaluation of corticotomyfacilitated orthodontics and piezocision in rapid canine retraction, Am J. Orthod. Dentofacial Orthop. 149 (4) (2016) 473-480

6. Al-Naoum F, Hajeer MY, Al-Jundi A. Does alveolar corticotomy accelerate orthodontic tooth movement when retracting upper canines? A split-mouth design randomized controlled trial. J Oral Maxillofac Surg. 2014;72:1880-9.

7. Bhattacharya P, Bhattacharya H, Anjum A, Bhandari R, Agarwal DK, Gupta A, et al. Assessment of Corticotomy Facilitated Tooth Movement and Changes in Alveolar Bone Thickness - A CT Scan Study. J Clin Diagn Res. 2014;8:ZC26-30.

8. Farid KA, Eid A., Kaddah MA, Elsharaby FA. The effect of combined corticotomy and low level laser therapy on the rate of orthodontic tooth movement: split mouth randomized clinical trial. Laser Ther. 2019; 28(4): 275-283.

9. Mahmoudzadeh M, Poormoradi B, Alijani S, Farhadian M Kazemisaleh A. Efficacy of $\mathrm{Er}, \mathrm{Cr}$ laser incision corticotomy in rapid maxillary canine retraction: a split-mouth randomized clinical trial. J Lasers Med Sci. 2020;11(4):442-449.

10. Omidkhoda M, Radvar M, Azizi M, Dehghani M. Evaluating the Efficacy of a Modified Piezo-Puncture Method on the Rate of Tooth Movement in Orthodontic Patients: A Clinical Study. Turk J Orthod 2020; 33(1): 13-20.

11. N.H. Abbas, N.E. Sabet, I.T. Hassan, Evaluation of corticotomyfacilitated orthodontics and piezocision in rapid canine retraction, Am. J. Orthod. Dentofacial Orthop. 149 (4) (2016) 473-480

12. Alfawal, Alaa M. H. et al. Evaluation of piezocision and laserassisted flapless corticotomy in the acceleration of canine retraction a randomized controlled trialHead \& Face Medicine (2018) 14:4 https:// doi.org/10.1186/s13005-018-0161-9 ную технику ретракции. Для уменьшение потери анкоража рекомендуется использовать ортодонтические минивинты, небный бюгель, проводить кортикотомию стенок лунки удаленного зуба и ретракцию с помощью эластической цепочки.

Поскольку ортодонтическое лечение проходят и взрослые пациенты, проведение аналогичных исследований было бы актуальным для возрастной группы старше 30 лет, с целью определения оптимальной методики для уменьшения сроков лечения и снижения риска развития побочных эффектов.

13. Aboul-Ela SM, El-Beialy AR, El-Sayed KM, Selim EM, ElMangoury NH, Mostafa YA. Miniscrew implant-supported maxillary canine retraction with and without corticotomy-facilitated orthodontics. Am J of Orthod Dentofacial Orthop. 2011;139:252-9.

14. Baeshen Hosam Ali The Effect of Partial Corticotomy on the Rate of Maxillary Canine Retraction: Clinical and Radiographic Study Molecules 2020, 25, 4837;

15. Hatrom AA, Zawawi KH, Al-Ali RM, Sabban MH, Zahid MT, Ghassan A. Al-Turki ; Ali H. Hassan. Effect of piezocision corticotomy on en-masse retraction: A randomized controlled trial. Angle Orthod (2020) 90 (5): 648-654.

16. Kumar N, Prashantha GS, Raikar S, Ranganath K, Mathew S, Nambiar S. Dento-Alveolar Distraction Osteogenesis for rapid Orthodontic Canine Retraction. J Int Oral Health 2013; 5(6):31-41.

17. Frost HM. The regional acceleratory phenomenon: a review. Henry Ford Hosp Med J. 1983;31:3-9.

18. Jahanbakhshi MR, Motamedi AM, Feizbakhsh M, Mogharehabed A. The effect of buccal corticotomy on accelerating orthodontic tooth movement of maxillary canine. Dent Res J 2016;13:303-8.

19. Baloul SS, Gerstenfeld LC, Morgan EF, Carvalho RS, Van Dyke TE, Kantarci A. Mechanism of action and morphologic changes in the alveolar bone in response to selective alveolar decortication-facilitated tooth movement. Am J Orthod Dentofac Orthop. 2011;139:S83-101.

20. Teixeira CC, Khoo E, Tran J, Chartres I, Liu Y, Thant LM, et al. Cytokine expression and accelerated tooth movement. J Dent Res. 2010;89:1135-41.

21. Fischer TJ. Orthodontic treatment acceleration with corticotomyassisted exposure of palatally impacted canines. Angle Orthod. 2007;77:417-20.)

22. Bermell-Baviera A, Bellot-Arcís C, Montiel-Company JM Almerich-Silla JM. Effects of mandibular advancement surgery on the temporomandibular joint and muscular and articular adaptive changes-a systematic review. Int J Oral Maxillofac Surg. 2016;45(5):545-52.

23. Agrawal, A.A.; Kolte, A.P.; Kolte, R.A.; Vaswani, V.; Shenoy, U.; Rathi, P. Comparative CBCT analysis of the changes in buccal bone morphology.2019.

Saudi Dental Journal 31(1): 58-65

24. Aksakalli S, Calik B, Kara B, Ezirganli S. Accelerated tooth movement with piezocision and its periodontal-transversal effects in patients with class II malocclusion. Angle Orthod. 2016;86:59-65.

25. Leethanakul C, Kanokkulchai S, Pongpanich S, Leepong N, Charoemratrote $\mathrm{C}$. Interseptal bone reduction on the rate of maxillary canine retraction. Angle Orthod. 2014;84:839-45.

\section{ИНФОРМАЦИЯ ОБ АВТОРАХ:}

Хабадзе 3.C. ${ }^{1}$ - к.м.н., доцент кафедры Терапевтической стоматологии, ORCID ID: 0000-0002-7257-5503.

Балашова M.E. ${ }^{2}$ - ординатор.

${ }^{1}$ Федеральное государственное автономное образовательное учреждение высшего образования «Российский университет дружбы народов», Москва, Россия

${ }^{2}$ Федеральное государственное бюджетное учреждение «Национальный медицинский исследовательский центр стоматологии и челюстно-лицевой хирургии» Министерства здравоохранения Российской Федерации, Москва, Россия

\section{AUTHOR INFORMATION:}

Z.S. Khabadze ${ }^{1}$ - Ph.D., Associate Professor, Department of Therapeutic Dentistry ORCID: 0000-0002-7257-5503

M.E. Balashova ${ }^{2}$ - resident student.

1"Peoples' Friendship University of Russia" (RUDN University), Moscow, Russia

${ }^{2}$ National Medical Research Center of Dentistry and Oral and Maxillofacial Surgery, Moscow, Russia

Координаты для связи с авторами / Coordinates for communication with authors: Хабадзе 3.C. / Z.S Khabadze, E-mail: dr.zura@mail.ru 